\title{
CONSERVATIVE ENERGY DISCRETIZATION OF BOLTZMANN COLLISION OPERATOR
}

\author{
BY \\ L. PREZIOSI AND L. RONDONI
}

Dipartimento di Matematica, Politecnico di Torino, Corso Duca degli Abruzzi 24, Torino, Italy

\begin{abstract}
The paper deduces a kinetic model obtained introducing a discretization of the Boltzmann equation based on an equispaced distribution of allowed particle energies. The model obtained is a system of integro-differential equations with integration over suitable angular variables: one over the portion of the unit sphere between two parallels symmetric with respect to the equatorial plane perpendicular to the velocity of the field particle, and one over a unit circle. The model preserves mass, momentum and energy. Furthermore, there exists an $H$-functional describing trend toward an equilibrium state described by a Gaussian distribution. Particular attention is paid to the identification of a criterion which indicates the values of the discretization parameters.
\end{abstract}

1. Introduction. The Boltzmann equation is the classical model used to describe the dynamics of rarefied gases. In principle, it is able to provide a quite accurate description of a physical system that is characterized by a huge number of collisions governed by classical mechanics. Unfortunately, from a numerical point of view, it is very hard to exploit all the capability of the model and all the physical information it contains.

This is essentially related to the structure of the collision term, a five-fold integral that is very expensive to be computed. Furthermore, this term needs to be evaluated with great precision, because all the mechanical properties characterizing particle collisions, e.g., conservation of mass, momentum, and energy, are included in it.

In order to tackle the multiple integration with a not too large number of operations, most of the numerical codes that integrate Boltzmann-like models make use of MonteCarlo procedures. Then, usually, in order to preserve mass, momentum, and energy, the solution is suitably corrected at each time step $[1,2]$.

On the other hand, very recently, several authors [3]-[8] focused their attention on the possibility of handling the integration in a different way. They aimed at identifying discretization procedures that lead to models possessing most of the properties characteristic of the Boltzmann equation, namely, conservation of mass, momentum, and

Received December 12, 1997.

1991 Mathematics Subject Classification. Primary 76P05.

Key words and phrases. Rarefied gas dynamics, Boltzmann equation. 
energy, validity of an $\mathbf{H}$-theorem, and stationary states described by Gaussian distributions. The determination of intrinsically conservative models is particularly important, because a lack of conservation, even if very small, may lead to serious consequences on the integration of the equation.

In particular, in [3-7] the velocity space is discretized in a uniform cubic lattice. Buet $[3,4]$ achieves the objective stated above using an infinite lattice, approximating the distribution function by piecewise constant functions, and through a not so clear definition of the transition rates. A finite cubic lattice is then used to describe the evolution of several problems of fluid-dynamic interest. Bobylev, Palczewski, and Schneider [5] focus on the number of intersections between the cubic lattice and the spheres having the relative velocity as diameter, showing that the number of intersections goes to infinity and tends to be uniformly distributed on the sphere. This allows the authors to prove the consistency of the quadrature rule they use for evaluating the collision term. However, the estimated rate of convergence is so small that a huge number of lattice points is needed to approximate the collision integral with a satisfactory precision. Rogier and Schneider [6] give a quadrature rule to approximate the collision integral of the planar Boltzmann equation, which yields an error that goes like $\log n / \sqrt{n}$ (where $n$ is the number of lattice points). Tcheremissine [7] achieves a conservative evaluation of the collision integral by a decomposition of each contribution to the gain term in a pair of contributions evaluated in the nearest points of the grid.

In [8] the Boltzmann equation is, instead, discretized using polar coordinates. The introduction of a suitable discretization of velocity moduli and of a suitable discretization procedure allows the authors to deduce a model in which the collision operator is a sum of integrals over finite domains. These are the cartesian product of a unit circle and the portion of the unit sphere between two parallels symmetric with respect to the equatorial plane perpendicular to the velocity of the field particle. One can then exploit the fact that integration over spherical domains can be performed with good precision and small computational effort. The resulting kinetic model preserves mass, momentum, and energy. Furthermore, there exists an $H$-functional describing trend toward an equilibrium described by a Gaussian distribution.

This paper considers particle velocities as defined through their energy and velocity direction. Discretizing the range of allowed energies in an equispaced fashion, and introducing a suitable discretization procedure, a model possessing the just-mentioned properties is deduced.

In particular, the paper focuses on the identification of the parameters involved in the discretization. This is done on the criterion that the spurious terms, which are present in the Euler equations related to this discretized model, are as small as requested by the application. In fact, as already mentioned in [8], whenever the velocity space is limited to a bounded subset (which is then discretized in order to be handled numerically) Boltzmann-like models lose their Galilean invariance. This is reflected into the introduction of two spurious terms in the related Euler equations which, however, tend rapidly to zero as both the diameter of the bounded subset and the number of discretized energies tend to infinity. 
The paper develops in four sections and an Appendix. After this introduction, Sec. 2 places the procedure in the frame of the splitting algorithm [9], whose convergence theorem has been recently proved by Desvillettes and Mischler [10], so that the paper can focus only on the collisional step, the most expensive from the computational viewpoint. The same section will also report some basic observations on the energy formulation of the model, that is, considering particles as characterized by their energy and velocity direction. Details on the collision dynamics in the energy formulation can then be found in the Appendix.

Section 3 deals with the necessity, from the computational point of view, of delimiting the range of allowed energy of the particles to a finite interval, with the criterion for the identification of such a range, and with the already-mentioned consequences of this unavoidable limitation.

Finally, in Sec. 4, the energy interval is partitioned in $n$ sub-intervals, and the kinetic model is deduced. It is found that

1. The model preserves mass, momentum and energy.

2. The modelling procedure is constructive, so that it is possible to obtain estimates on the "distance" between the discretized collision operator and the continuous Boltzmann operator, i.e., consistency of the quadrature rule.

3. There exists an $H$-functional describing trend towards an equilibrium described by a Gaussian distribution.

4. The fluid-dynamic limit related to the discretized kinetic model tends rapidly towards the usual Euler equations with an isotropic pressure tensor and vanishing heat flux when the number of allowed energies grows to infinity and the discretization interval for the particle energies becomes $\mathbb{R}_{+}$. This last step is obtained by carefully studying the behavior of the spurious terms that originate from the discretization, and that make possible the identification of a discretization procedure.

2. Splitting and energy formulation. In kinetic theory, the behaviour of a neutral, rarefied gas is usually described through the evolution of a distribution function, $f:[0, T] \times \mathbb{R}^{3} \times \mathbb{R}^{3} \rightarrow \mathbb{R}_{+}$, which satisfies the Boltzmann equation

$$
\left\{\begin{array}{l}
\frac{\partial f}{\partial t}=\mathcal{A}(f)+\mathcal{Q}(f, f) \\
f(0, \mathbf{x}, \mathbf{v})=f_{0}(\mathbf{x}, \mathbf{v})
\end{array}\right.
$$

for all times $t \in[0, T]$, for some $T>0$, where

$$
\mathcal{A}(f)=-\mathbf{v} \cdot \nabla_{x} f
$$

is a linear differential operator and

$$
\mathcal{Q}(f, f)=\int_{\mathbb{R}^{3}} \int_{S_{2}} B(\mathbf{g}, \hat{\mathbf{n}})\left[f\left(t, \mathbf{x}, \mathbf{v}^{\prime}\right) f\left(t, \mathbf{x}, \mathbf{v}_{*}^{\prime}\right)-f(t, \mathbf{x}, \mathbf{v}) f\left(t, \mathbf{x}, \mathbf{v}_{*}\right)\right] d \hat{\mathbf{n}} d \mathbf{v}_{*}
$$


is a nonlinear integral operator, which is rather heavy to evaluate numerically, since it consists of a five-fold integral with several important macroscopic properties.

In (2.3) $\mathbf{g}=\mathbf{v}_{*}-\mathbf{v}$ is the relative velocity of colliding particles, $B$ is a collision kernel depending on the interaction potential, and the rule for collision between two particles is expressed by

$$
\begin{aligned}
& \mathbf{v}^{\prime}=\mathbf{v}+(\mathbf{g} \cdot \hat{\mathbf{n}}) \hat{\mathbf{n}}=\frac{\mathbf{R}}{2}+\frac{g}{2} \hat{\mathbf{g}}^{\prime} \\
& \mathbf{v}_{*}^{\prime}=\mathbf{v}_{*}-(\mathbf{g} \cdot \hat{\mathbf{n}}) \hat{\mathbf{n}}=\frac{\mathbf{R}}{2}-\frac{g}{2} \hat{\mathbf{g}}^{\prime}
\end{aligned}
$$

where

$$
\begin{gathered}
\mathbf{R} \stackrel{\text { def }}{\equiv} \mathbf{v}+\mathbf{v}_{*}=\mathbf{v}^{\prime}+\mathbf{v}_{*}^{\prime}, \\
g \stackrel{\text { def }}{\equiv}\left|\mathbf{v}_{*}-\mathbf{v}\right|=\left|\mathbf{v}_{*}^{\prime}-\mathbf{v}^{\prime}\right|,
\end{gathered}
$$

and $\hat{\mathbf{n}}$ and $\hat{\mathbf{g}}^{\prime}$ are unit vectors that span $\mathbf{S}_{2}$. In particular, $\hat{\mathbf{g}}^{\prime}$ represents the direction of the relative velocity after collision.

In the following, particles are considered as characterized by their energy per unit mass, $e=v^{2} / 2$ (simply named energy for sake of brevity), and by their velocity direction $\widehat{\Omega}$. It is then useful to write

$$
g=\sqrt{2(E-s)}, \quad R \stackrel{\text { def }}{\equiv}|\mathbf{R}|=\sqrt{2(E+S)},
$$

where

$$
E \stackrel{\text { def }}{\equiv} e+e_{*}=e^{\prime}+e_{*}^{\prime}
$$

and

$$
S \stackrel{\text { def }}{\equiv} 2 \sqrt{e e_{*}} \widehat{\mathbf{\Omega}} \cdot \widehat{\mathbf{\Omega}}_{*}=2 \sqrt{e^{\prime} e_{*}^{\prime}} \widehat{\mathbf{\Omega}}^{\prime} \cdot \widehat{\boldsymbol{\Omega}}_{*}^{\prime}
$$

are, together with $\mathbf{R}$ and $g$ defined in (2.5) and (2.6), quantities that are invariant during collision.

One of the most important methods for the numerical solution of Eqs. (2.1) is the socalled "splitting algorithm", first applied by Temam [9] in the study of several evolution problems, like the Broadwell model. This method, when applied to the Boltzmann equation, consists in decoupling the collision contributions from the free streaming ones, and looking for iterative solutions of the following problems:

$$
\begin{gathered}
f_{n}^{0}(t=0)=\tilde{f}_{n}^{0}(t=0)=f_{0} ; \\
\begin{cases}\frac{\partial f_{n}^{k}}{\partial t}=\mathcal{A} f_{n}^{k}, & \text { for } k>0 ; \\
f_{n}^{k}\left(t_{k}\right)=\tilde{f}_{n}^{k-1}\left(t_{k}\right) & \end{cases}
\end{gathered}
$$




$$
\left\{\begin{array}{l}
\frac{\partial \tilde{f}_{n}^{k}}{\partial t}=\mathcal{Q} \tilde{f}_{n}^{k} \\
\tilde{f}_{n}^{k}\left(t_{k}\right)=f_{n}^{k}\left(t_{k+1}\right)
\end{array} \text { for } k>0 .\right.
$$

The convergence of the algorithm has been recently proven by Desvillettes and Mischler [10], under the same assumptions used by R. J. DiPerna and P.-L. Lions [11] for their existence results for the Boltzmann equation.

In the following we develop a procedure to approach the solution of the collision step for the splitting algorithm, Eqs. (2.12), where we switch from the description involving particle velocities to a description in terms of particle energies and velocity directions. As a matter of fact, the collisional step is the heaviest one from the numerical point of view, since it consists of the evaluation of a five-fold integral, which needs to be performed with great accuracy. Indeed, among other things, mass, momentum, and energy conservation during collisions depend on such an integral. The description of the collision dynamics using the energy formulation is given in the Appendix.

To understand the discretization procedure, which will be described in Secs. 3 and 4, it is important to observe that Eq. (2.9) implies a restriction acting either on the outgoing energy $e^{\prime}$, or on the angle between the incoming directions $\widehat{\boldsymbol{\Omega}} \cdot \widehat{\boldsymbol{\Omega}}_{*}$, considering the outgoing energies fixed.

This is a well-known fact when looked at from the classical point of view.

- Given the incoming energies $e, e_{*}$ and $\widehat{\boldsymbol{\Omega}} \cdot \widehat{\boldsymbol{\Omega}}_{*}$, the outgoing energies are confined inside the interval

$$
\mathcal{D}_{e^{\prime}}=\left[\frac{E}{2}-\frac{g R}{4}, \frac{E}{2}+\frac{g R}{4}\right],
$$

symmetrically with respect to its extrema.

For the deduction of the model that is proposed in Sec. 4, it is useful to look at the same restriction from a different point of view.

- Given the incoming energies $e$ and $e_{*}$, and an outgoing energy $e^{\prime}$ smaller than the largest energy the particle can possibly reach, $E=e+e_{*}\left(e_{*}^{\prime}\right.$ is then determined by the conservation of energy, i.e., $\left.e_{*}^{\prime}=E-e^{\prime}\right)$, there is a limited range for the angle between the pre-collisional velocity directions which can yield a post-collisional energy equal to $e^{\prime}$. This can be expressed as

$$
\left(\widehat{\boldsymbol{\Omega}} \cdot \widehat{\boldsymbol{\Omega}}_{*}\right)^{2} \leq \lambda \stackrel{\text { def }}{\equiv} \frac{e^{\prime} e_{*}^{\prime}}{e e_{*}} .
$$

It is then useful to define the domain

$$
\mathcal{D}_{\widehat{\boldsymbol{\Omega}}_{*}}=\left\{\widehat{\boldsymbol{\Omega}}_{*}:\left(\widehat{\boldsymbol{\Omega}} \cdot \widehat{\boldsymbol{\Omega}}_{*}\right)^{2} \leq \lambda=\frac{e^{\prime} e_{*}^{\prime}}{e e_{*}}\right\},
$$

which represents either the entire unit sphere $\mathbf{S}_{2}$, if $e^{\prime} e_{*}^{\prime} \geq e e_{*}$ or, taking $\widehat{\boldsymbol{\Omega}}$ as polar axis, that part between two parallels symmetric with respect to the equator, as shown in Fig. 1. 


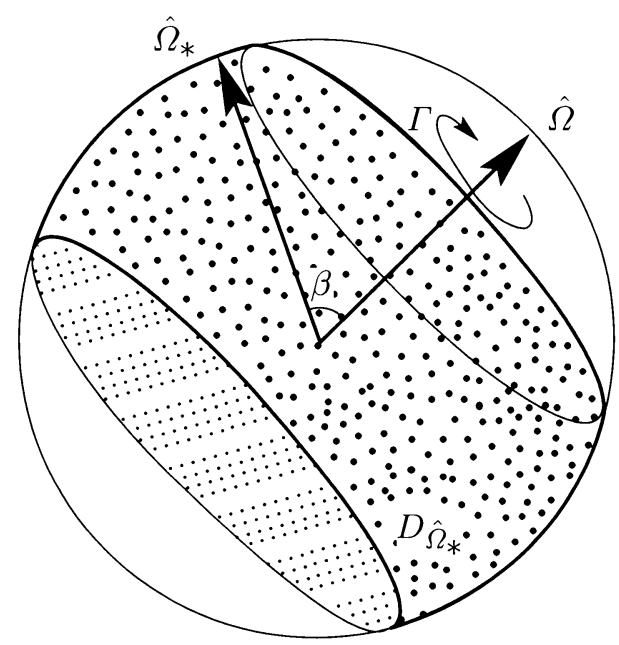

FIG. 1. The domain $\mathcal{D}_{\hat{\Omega}_{*}}$

3. Identification of the energy interval. The very first step of any discretization procedure is to define in the velocity space a limited subset containing all allowed velocities. In [8] it has been observed that a consequence of this step is the appearance of two spurious terms in the momentum and energy Euler equations related to the Boltzmann equation, namely an extra pressure tensor, in the form of an extra Reynolds stress term, and an extra heat flux term, in the form of an extra pressure.

The presence of these terms is directly related to the introduction of the "boundary" in the velocity space which corresponds to an approximation of the original equations, which breaks the Galilean invariance characteristic both of the continuous Boltzmann equation, and of the related Euler equations. To clarify this statement, one can consider the viewpoint of the particle that sees (or feels) this artificial boundary as a reference point. Therefore, one cannot expect that the Euler equations related to any discretization scheme have an isotropic pressure tensor and a vanishing heat flux.

To be more specific, consider the conservation equations related to the continuous Boltzmann equation:

$$
\left\{\begin{array}{l}
\frac{\partial \rho}{\partial t}+\nabla \cdot(\rho \mathbf{U})=0 \\
\frac{\partial}{\partial t}(\rho \mathbf{U})+\nabla \cdot(\boldsymbol{\Pi}+\rho \mathbf{U} \otimes \mathbf{U})=\mathbf{0} \\
\frac{\partial}{\partial t}\left(\mathcal{E}+\frac{1}{2} \rho U^{2}\right)+\nabla \cdot\left[\mathbf{q}+\boldsymbol{\Pi} \mathbf{U}+\left(\mathcal{E}+\frac{1}{2} \rho U^{2}\right) \mathbf{U}\right]=0
\end{array}\right.
$$

where $\rho$ is the mass density, $\mathbf{U}$ is the drift velocity and $U$ its modulus, $\mathcal{E}$ is the internal energy, $\boldsymbol{\Pi}$ is the pressure tensor, and $\mathbf{q}$ is the heat flux.

Referring to [8] for further details, we compute the macroscopic quantities and, in particular, the pressure tensor and the heat flux at Maxwellian equilibrium, considered as a function of energy and velocity direction,

$$
f\left(t, \mathbf{x}, e, \widehat{\boldsymbol{\Omega}}^{\prime}\right)=A \exp [\sqrt{b e} \widehat{\mathbf{U}} \cdot \widehat{\boldsymbol{\Omega}}-c e], \quad \text { with } b=\frac{1}{2}\left(\frac{3 \rho U}{\mathcal{E}}\right)^{2}, \quad c=\frac{3 \rho}{2 \mathcal{E}},
$$


and where $\widehat{\mathbf{U}}=\mathbf{U} / U$ is the direction of the drift velocity. One can then write the pressure tensor and the heat flux as

$$
\boldsymbol{\Pi}=\Pi\left(\mathbf{I}+\eta_{\pi} \widehat{\boldsymbol{\Omega}} \otimes \widehat{\mathbf{\Omega}}\right), \quad \mathbf{q}=\Pi\left(\varepsilon_{q}-\eta_{\pi}\right) \mathbf{U}
$$

where

$\eta_{\pi}=\frac{\int_{\mathbb{R}_{+}^{2}} e^{-c\left(e+e^{\prime}\right)}\left[(b e+2) \sinh \sqrt{b e} \sinh \sqrt{b e^{\prime}}-\sqrt{b e} \cosh \sqrt{b e} \sinh \sqrt{b e^{\prime}}-b \sqrt{e e^{\prime}} \cosh \sqrt{b e} \cosh \sqrt{b e^{\prime}}\right] d e d e^{\prime}}{\int_{\mathbb{R}_{+}^{2}} e^{-c\left(e+e^{\prime}\right)}(\sqrt{b e} \cosh \sqrt{b e}-\sinh \sqrt{b e}) \sinh \sqrt{b e^{\prime}} d e d e^{\prime}}$,

$$
\varepsilon_{q}=\frac{\int_{\mathbb{R}_{+}^{2}} \sqrt{b e}\left(e-e^{\prime}\right) e^{-c\left(e+e^{\prime}\right)} \cosh \sqrt{b e} \sinh \sqrt{b e^{\prime}} d e d e^{\prime}}{2\left[\int_{\mathbb{R}_{+}} e^{-c e}(\sqrt{b e} \cosh \sqrt{b e}-\sinh \sqrt{b e}) d e\right]^{2}} b-1,
$$

and the Euler equations as

$$
\left\{\begin{array}{l}
\frac{\partial \rho}{\partial t}+\nabla \cdot(\rho \mathbf{U})=0 \\
\frac{\partial}{\partial t}(\rho \mathbf{U})+\nabla \cdot\left[\left(1+\varepsilon_{\pi}\right) \rho \mathbf{U} \otimes \mathbf{U}\right]+\nabla \Pi=\mathbf{0} \\
\frac{\partial}{\partial t}\left(\mathcal{E}+\frac{1}{2} \rho U^{2}\right)+\nabla \cdot\left\{\left[\left(1+\varepsilon_{q}\right) \Pi+\mathcal{E}+\frac{1}{2} \rho U^{2}\right] \mathbf{U}\right\}=0
\end{array}\right.
$$

where

$\varepsilon_{\pi}=\frac{\int_{\mathbb{R}_{+}^{2}} e^{-c\left(e+e^{\prime}\right)}\left[(b e+2) \sinh \sqrt{b e} \sinh \sqrt{b e^{\prime}}-\sqrt{b e} \cosh \sqrt{b e} \sinh \sqrt{b e^{\prime}}-b \sqrt{e e^{\prime}} \cosh \sqrt{b e} \cosh \sqrt{b e^{\prime}}\right] d e d e^{\prime}}{\left[\int_{\mathbb{R}_{+}} e^{-c e}(\sqrt{b e} \cosh \sqrt{b e}-\sinh \sqrt{b e}) d e\right]^{2}}$.

The integrals in $(3.4,3.5,3.7)$ can be computed analytically, and give $\eta_{\pi}=\varepsilon_{\pi}=$ $\varepsilon_{q}=0$, corresponding to an isotropic pressure tensor, a vanishing heat flux, and no extra terms in the Euler equations (3.6), as it must be. If, instead, the integrals in $(3.4,3.5$, 3.7) are approximated by computing them only over the finite interval $\left[0, e_{M}\right)$, a lengthy calculation gives

$$
\begin{aligned}
& \eta_{\pi}\left(r, 0, \tilde{e}_{M}\right)=\frac{2\left[\mathcal{N}_{\pi}\left(r, \tilde{e}_{M}\right)-\widetilde{\mathcal{E}}\left(r, \tilde{e}_{M}\right) \mathcal{M}_{\pi}\left(r, \tilde{e}_{M}\right)\right]}{\mathcal{D}_{\pi}\left(r, \tilde{e}_{M}\right) \mathcal{D}_{q}\left(r, \tilde{e}_{M}\right)}, \\
& \varepsilon_{\pi}\left(r, 0, \tilde{e}_{M}\right)=\frac{2\left[\mathcal{N}_{\pi}\left(r, \tilde{e}_{M}\right)-\widetilde{\mathcal{E}}\left(r, \tilde{e}_{M}\right) \mathcal{M}_{\pi}\left(r, \tilde{e}_{M}\right)\right]}{\mathcal{D}_{q}^{2}\left(r, \tilde{e}_{M}\right)} \\
& \varepsilon_{q}\left(r, 0, \tilde{e}_{M}\right)=\frac{2\left[\mathcal{N}_{q}\left(r, \tilde{e}_{M}\right)-\widetilde{\mathcal{E}}\left(r, \tilde{e}_{M}\right) \mathcal{M}_{q}\left(r, \tilde{e}_{M}\right)\right]}{\mathcal{D}_{q}^{2}\left(r, \tilde{e}_{M}\right)}
\end{aligned}
$$


where

$$
\begin{aligned}
& \mathcal{N}_{\pi}(r, \tilde{e})= 2 e^{-r \tilde{e}^{2}}\left[(r+2) \sinh ^{2} r \tilde{e}-(r+1) r \tilde{e} \sinh r \tilde{e} \cosh r \tilde{e}-r^{2} \tilde{e}^{2}\right], \\
& \mathcal{M}_{\pi}(r, \tilde{e})= e^{-r \tilde{e}^{2} / 2}\left[\left(r+3+r^{2} \tilde{e}^{2}\right) \sinh r \tilde{e}-(r+3) r \tilde{e} \cosh r \tilde{e}\right], \\
& \mathcal{N}_{q}(r, \tilde{e})=2 e^{-r \tilde{e}^{2}}\left\{\left[r^{2}+4 r-2-(r+2) r^{2} \tilde{e}^{2}\right] \sinh ^{2} r \tilde{e}\right. \\
&\left.\quad \quad-\left(r^{2}+3 r-4\right) r \tilde{e} \sinh r \tilde{e} \cosh r \tilde{e}-(r+2) r^{2} \tilde{e}^{2}\right\}, \\
& \mathcal{M}_{q}(r, \tilde{e})=e^{-r \tilde{e}^{2} / 2}\left[r(r+5)-r^{2} \tilde{e}^{2}\right](\sinh r \tilde{e}-r \tilde{e} \cosh r \tilde{e}), \\
& \mathcal{D}_{\pi}(r, \tilde{e})=\tilde{\mathcal{E}}(r, \tilde{e})-2 e^{-r \tilde{e}^{2} / 2} \sinh r \tilde{e}, \\
& \mathcal{D}_{q}(r, \tilde{e})=r \widetilde{\mathcal{E}}(r, \tilde{e})-2 e^{-r \tilde{e}^{2} / 2}[(r-1) \sinh r \tilde{e}+r \tilde{e} \cosh r \tilde{e}], \\
& \widetilde{\mathcal{E}}(r, \tilde{e})=\sqrt{\frac{\pi r}{2}} e^{r / 2}\left[\operatorname{Erf}\left(\sqrt{\frac{r}{2}}(1+\tilde{e})\right)-\operatorname{Erf}\left(\sqrt{\frac{r}{2}}(1-\tilde{e})\right)\right],
\end{aligned}
$$

$\operatorname{Erf}(\cdot)$ is the error function, and

$$
r=\frac{3 \rho U^{2}}{2 \mathcal{E}}, \quad \tilde{e}=\frac{\sqrt{2 e}}{U}
$$

The zero in the argument of $\eta_{\pi}, \varepsilon_{\pi}$, and $\varepsilon_{q}$ in $(3.8-3.10)$ is due to our choice $\tilde{e}_{m}=0$ as the lowest end of the interval $\left[0, \tilde{e}_{M}\right)$. In general, one can use $\left[\tilde{e}_{m}, \tilde{e}_{M}\right)$ as in Eqs. (3.14-3.16) below.

The terms in (3.8-3.10) do not vanish, and therefore the pressure tensor is not isotropic, the heat flux does not vanish, and the two spurious terms appearing in the Euler equation give a nonzero contribution. However, it can be seen that, as $\tilde{e}_{M}$ goes to infinity, $\eta_{\pi}, \varepsilon_{\pi}$, and $\varepsilon_{q}$ all go exponentially to zero. Of course, all macroscopic variables in (3.6) are consistently defined over the bounded subset $\left[0, e_{M}\right)$.

The introduction of a lower bound, say $e_{m}$, gives further contributions to the spurious terms which go to zero as $e_{m}^{3 / 2}$. In this case, $\eta_{\pi}, \varepsilon_{\pi}$, and $\varepsilon_{q}$ can be written as

$$
\begin{aligned}
& \eta_{\pi}\left(r, \tilde{e}_{m}, \tilde{e}_{M}\right) \\
& \quad=\frac{2\left\{\mathcal{N}_{\pi}\left(r, \tilde{e}_{M}\right)+\mathcal{N}_{\pi}\left(r, \tilde{e}_{m}\right)-\left[\widetilde{\mathcal{E}}\left(r, \tilde{e}_{M}\right)-\widetilde{\mathcal{E}}\left(r, \tilde{e}_{m}\right)\right]\left[\mathcal{M}_{\pi}\left(r, \tilde{e}_{M}\right)+\mathcal{M}_{\pi}\left(r, \tilde{e}_{m}\right)\right]+\mathcal{N}_{\pi}^{\prime}\left(r, \tilde{e}_{m}, \tilde{e}_{M}\right)\right\}}{\left[\mathcal{D}_{\pi}\left(r, \tilde{e}_{M}\right)-\mathcal{D}_{\pi}\left(r, \tilde{e}_{m}\right)\right]\left[\mathcal{D}_{q}\left(r, \tilde{e}_{M}\right)-\mathcal{D}_{q}\left(r, \tilde{e}_{m}\right)\right]}, \\
& \varepsilon_{\pi}\left(r, \tilde{e}_{m}, \tilde{e}_{M}\right) \\
& \quad=\frac{2\left\{\mathcal{N}_{\pi}\left(r, \tilde{e}_{M}\right)+\mathcal{N}_{\pi}\left(r, \tilde{e}_{m}\right)-\left[\widetilde{\mathcal{E}}\left(r, \tilde{e}_{M}\right)-\widetilde{\mathcal{E}}\left(r, \tilde{e}_{m}\right)\right]\left[\mathcal{M}_{\pi}\left(r, \tilde{e}_{M}\right)+\mathcal{M}_{\pi}\left(r, \tilde{e}_{m}\right)\right]+\mathcal{N}_{\pi}^{\prime}\left(r, \tilde{e}_{m}, \tilde{e}_{M}\right)\right\}}{\left[\mathcal{D}_{q}\left(r, \tilde{e}_{M}\right)-\mathcal{D}_{q}\left(r, \tilde{e}_{m}\right)\right]^{2}}, \\
& \varepsilon_{q}\left(r, \tilde{e}_{m}, \tilde{e}_{M}\right) \\
& \quad=\frac{2\left\{\mathcal{N}_{q}\left(r, \tilde{e}_{M}\right)+\mathcal{N}_{q}\left(r, \tilde{e}_{m}\right)-\left[\widetilde{\mathcal{E}}\left(r, \tilde{e}_{M}\right)-\widetilde{\mathcal{E}}\left(r, \tilde{e}_{m}\right)\right]\left[\mathcal{M}_{q}\left(r, \tilde{e}_{M}\right)+\mathcal{M}_{q}\left(r, \tilde{e}_{m}\right)\right]+\mathcal{N}_{q}^{\prime}\left(r, \tilde{e}_{m}, \tilde{e}_{M}\right)\right\}}{\left[\mathcal{D}_{q}\left(r, \tilde{e}_{M}\right)-\mathcal{D}_{q}\left(r, \tilde{e}_{m}\right)\right]^{2}},
\end{aligned}
$$


where

$$
\begin{aligned}
& \mathcal{N}_{\pi}^{\prime}\left(r, \tilde{e}_{m}, \tilde{e}_{M}\right)=e^{-r\left(\tilde{e}_{m}^{2}+\tilde{e}_{M}^{2}\right) / 2}\left\{-4(r+2) \sinh r \tilde{e}_{m} \sinh r \tilde{e}_{M}\right. \\
&+(r+1) r\left[\left(\tilde{e}_{m}+\tilde{e}_{M}\right) \sinh r\left(\tilde{e}_{m}+\tilde{e}_{M}\right)-\left(\tilde{e}_{M}-\tilde{e}_{m}\right) \sinh r\left(\tilde{e}_{M}-\tilde{e}_{m}\right)\right] \\
&\left.+r^{2}\left[\left(\tilde{e}_{m}+\tilde{e}_{M}\right)^{2} \cosh r\left(\tilde{e}_{M}-\tilde{e}_{m}\right)-\left(\tilde{e}_{M}-\tilde{e}_{m}\right)^{2} \cosh r\left(\tilde{e}_{M}+\tilde{e}_{m}\right)\right]\right\}
\end{aligned}
$$

$$
\begin{aligned}
\mathcal{N}_{q}^{\prime}\left(r, \tilde{e}_{m}, \tilde{e}_{M}\right)=e^{-} & r\left(\tilde{e}_{m}^{2}+\tilde{e}_{M}^{2}\right) / 2\left\{-4\left(r^{2}+4 r-2\right) \sinh r \tilde{e}_{m} \sinh r \tilde{e}_{M}\right. \\
& +4(r+2) r^{2} \tilde{e}_{m} \tilde{e}_{M} \cosh r \tilde{e}_{m} \cosh r \tilde{e}_{M} \\
& +\left(r^{2}+3 r-4\right) r\left[\left(\tilde{e}_{m}+\tilde{e}_{M}\right) \sinh r\left(\tilde{e}_{m}+\tilde{e}_{M}\right)\right. \\
& \left.-\left(\tilde{e}_{M}-\tilde{e}_{m}\right) \sinh r\left(\tilde{e}_{M}-\tilde{e}_{m}\right)\right]+r^{3}\left[\left(\tilde{e}_{m}+\tilde{e}_{M}\right)^{2} \cosh r\left(\tilde{e}_{M}-\tilde{e}_{m}\right)\right. \\
& \left.\left.-\left(\tilde{e}_{M}-\tilde{e}_{m}\right)^{2} \cosh r\left(\tilde{e}_{M}+\tilde{e}_{m}\right)\right]\right\}
\end{aligned}
$$

and $\tilde{e}_{m}=\sqrt{2 e_{m}} / U$.

These estimates characterize the magnitude of the spurious terms introduced when the velocity space is confined to a sphere or a hollow sphere, and they provide us with a tool to control such terms. On these grounds, the following procedure for choosing $e_{m}$ and $e_{M}$ may be outlined.

1) Identify the range of internal energy and drift velocity characteristic of the flow to be described (and therefore $r$ ).

2) Set a desired accuracy $\bar{\varepsilon}$ for the spurious terms $\varepsilon_{\pi}$ (or $\eta_{\pi}$ ) and $\varepsilon_{q}$ appearing in the Euler equations (3.6) or in the definition of heat flux and pressure tensor, and define $\bar{\varepsilon}_{m}$ and $\bar{\varepsilon}_{M}$ such that $\bar{\varepsilon}_{b}=\bar{\varepsilon}_{M}+\bar{\varepsilon}_{m}<\bar{\varepsilon}$ (say, $\bar{\varepsilon}_{M}=\bar{\varepsilon}_{m}=\bar{\varepsilon} / 3$ ).

3) Set $\tilde{e}_{M}$ so that $\varepsilon_{\pi}$ (or $\eta_{\pi}$ ) and $\varepsilon_{q}$ in Eqs. (3.8-3.10) have absolute value smaller than $\bar{\varepsilon}_{M}$. In fact, for instance, Eq. (3.10) gives $\varepsilon_{q}=\varepsilon_{q}\left(r, 0, \tilde{e}_{M}\right)$, which (locally) defines $\tilde{\varepsilon}_{M}=\tilde{\varepsilon}_{M}^{q}\left(r, 0, \varepsilon_{q}\right)$. Therefore we can set

$$
\tilde{e}_{M}=\bar{e}_{M}\left(r, \bar{\varepsilon}_{M}\right) \stackrel{\text { def }}{\equiv} \max \left\{\tilde{e}_{M}^{\pi}\left(r, 0, \bar{\varepsilon}_{M}\right), \tilde{e}_{M}^{q}\left(r, 0, \bar{\varepsilon}_{M}\right)\right\}
$$

4) Having set $\tilde{e}_{M}$ according to (3.18), one can consider $\tilde{e}_{m}$ so that $\varepsilon_{\pi}$ (or $\eta_{\pi}$ ) and $\varepsilon_{q}$ in Eqs. (3.14-3.16) have absolute value smaller than $\bar{\varepsilon}_{b}=\bar{\varepsilon}_{m}+\bar{\varepsilon}_{M}$. In fact, for instance, Eq. (3.16) determines which values of $\tilde{e}_{m}$ yield $\varepsilon_{q}\left(r, \tilde{e}_{m}, \bar{\varepsilon}_{M}\left(r, \bar{\varepsilon}_{M}\right)\right)=\bar{\varepsilon}_{m}+\bar{\varepsilon}_{M}$, that is, $\tilde{e}_{m}=\tilde{e}_{m}^{q}\left(r, \bar{\varepsilon}_{m}+\bar{\varepsilon}_{M}, \bar{\varepsilon}_{M}\right)$. Therefore, if computationally convenient, one can set

$$
\tilde{e}_{m}=\bar{e}_{m}\left(r, \bar{\varepsilon}_{m}, \bar{\varepsilon}_{M}\right) \stackrel{\text { def }}{\equiv} \min \left\{\tilde{e}_{m}^{\pi}\left(r, \bar{\varepsilon}_{m}+\bar{\varepsilon}_{M}, \bar{\varepsilon}_{M}\right), \tilde{e}_{m}^{q}\left(r, \bar{\varepsilon}_{m}+\bar{\varepsilon}_{M}, \bar{\varepsilon}_{M}\right)\right\} \text {. }
$$

This procedure is used to plot Fig. 2 and to give Tables 1 and 2. In particular, Fig. 2 gives $\varepsilon_{\pi}$ and $\varepsilon_{q}$ as a function of $\tilde{e}_{M}$ at different $r$, Table 1 gives $\bar{e}_{M}\left(r, \frac{\bar{\varepsilon}}{3}\right)$, and Table 2 gives $\bar{e}_{m}\left(r, \frac{\bar{\varepsilon}}{3}, \frac{\bar{\varepsilon}}{3}\right)$ at fixed values of $r$ and overall accuracy $\bar{\varepsilon}$. 


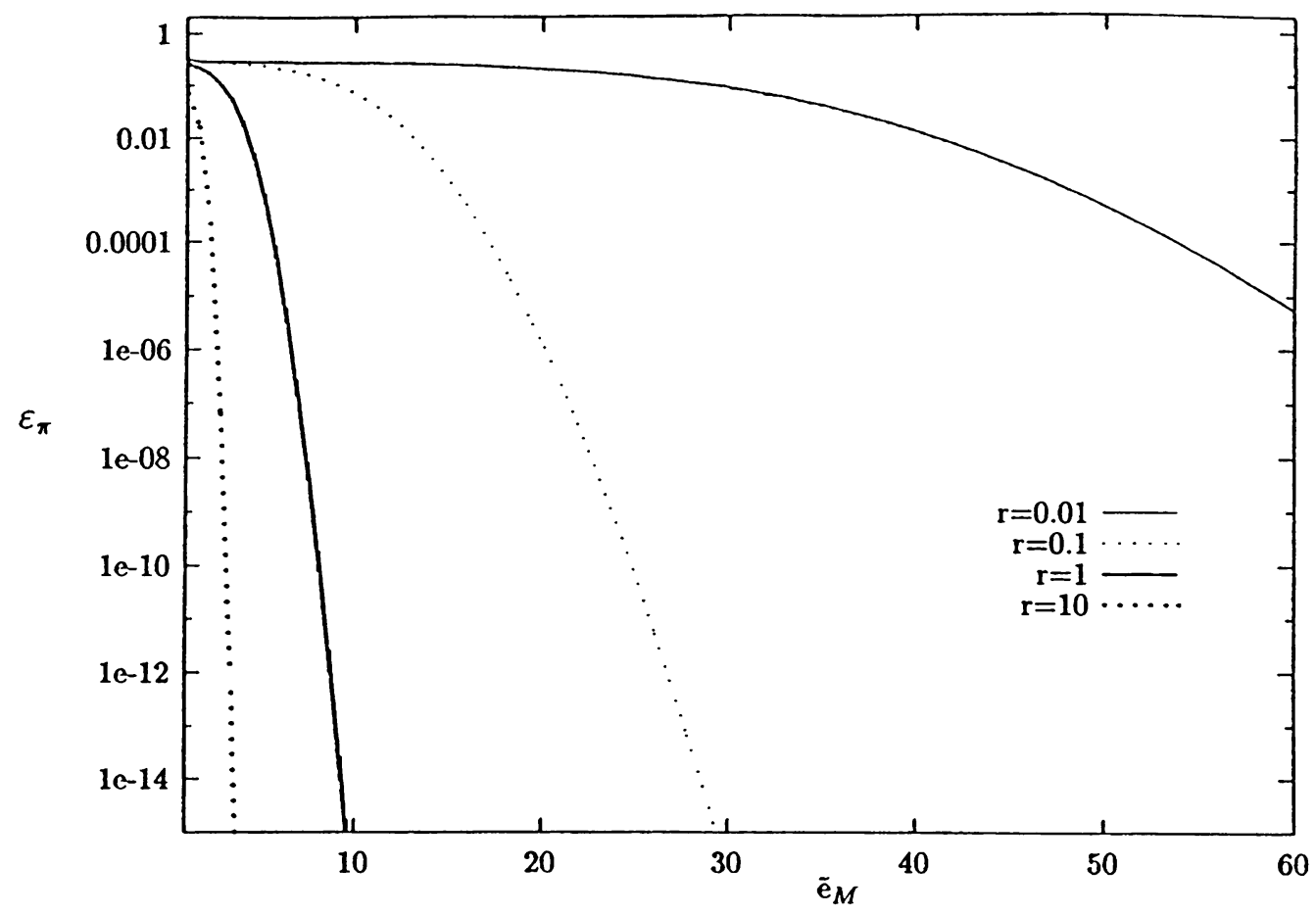

FIG. 2(a). Plot of $\varepsilon_{\pi}$ as a function of $\tilde{e}_{M}$ at different values of the expected macroscopic parameter $r=3 \rho U^{2} /(2 \varepsilon)$. The plot gives an indication of the value of the parameter $\tilde{e}_{M}$ to be chosen, in the discretization, so that the induced magnitude of the spurious terms is small enough.

\begin{tabular}{|c|c|c|c|c|}
\hline & $\begin{aligned} \bar{\varepsilon} & =10^{-1} \\
\bar{\varepsilon}_{M I} & =\frac{1}{3} 10^{-1}\end{aligned}$ & $\begin{array}{c}\bar{\varepsilon}=10^{-2} \\
\bar{\varepsilon}_{M I}=\frac{1}{3} 10^{-2}\end{array}$ & $\begin{array}{c}\bar{\varepsilon}=10^{-3} \\
\bar{\varepsilon}_{M I}=\frac{1}{3} 10^{-3}\end{array}$ & $\begin{array}{r}\bar{\varepsilon}=10^{-4} \\
\bar{\varepsilon}_{M}=\frac{1}{3} 10^{-4}\end{array}$ \\
\hline$r=10$ & 2.025 & 2.265 & 2.457 & 2.622 \\
\hline$r=1$ & 4.603 & 5.326 & 5.908 & 6.410 \\
\hline$r=0.1$ & 13.53 & 15.72 & 17.49 & 19.03 \\
\hline$r=0.01$ & 42.41 & 49.28 & 54.83 & 59.65 \\
\hline
\end{tabular}

TABLE 1. Values of $\bar{e}_{M}\left(r, \bar{\varepsilon}_{M}=\frac{\bar{\varepsilon}}{3}\right)$ defined in (3.18) at different values of the expected macroscopic parameter $r=3 \rho U^{2} /(2 \mathcal{E})$, and overall accuracy $\bar{\varepsilon}$.

It should be noticed that for physical reasons $e_{m}$ should be chosen smaller than $U^{2} / 2$, i.e., $\tilde{e}_{m}<1$. In Table 2 there are, however, some values of $r$ and $\bar{\varepsilon}$ for which the contribution related to the introduction of any $\tilde{e}_{m}<1$ is always less than $\bar{\varepsilon} / 3$. 


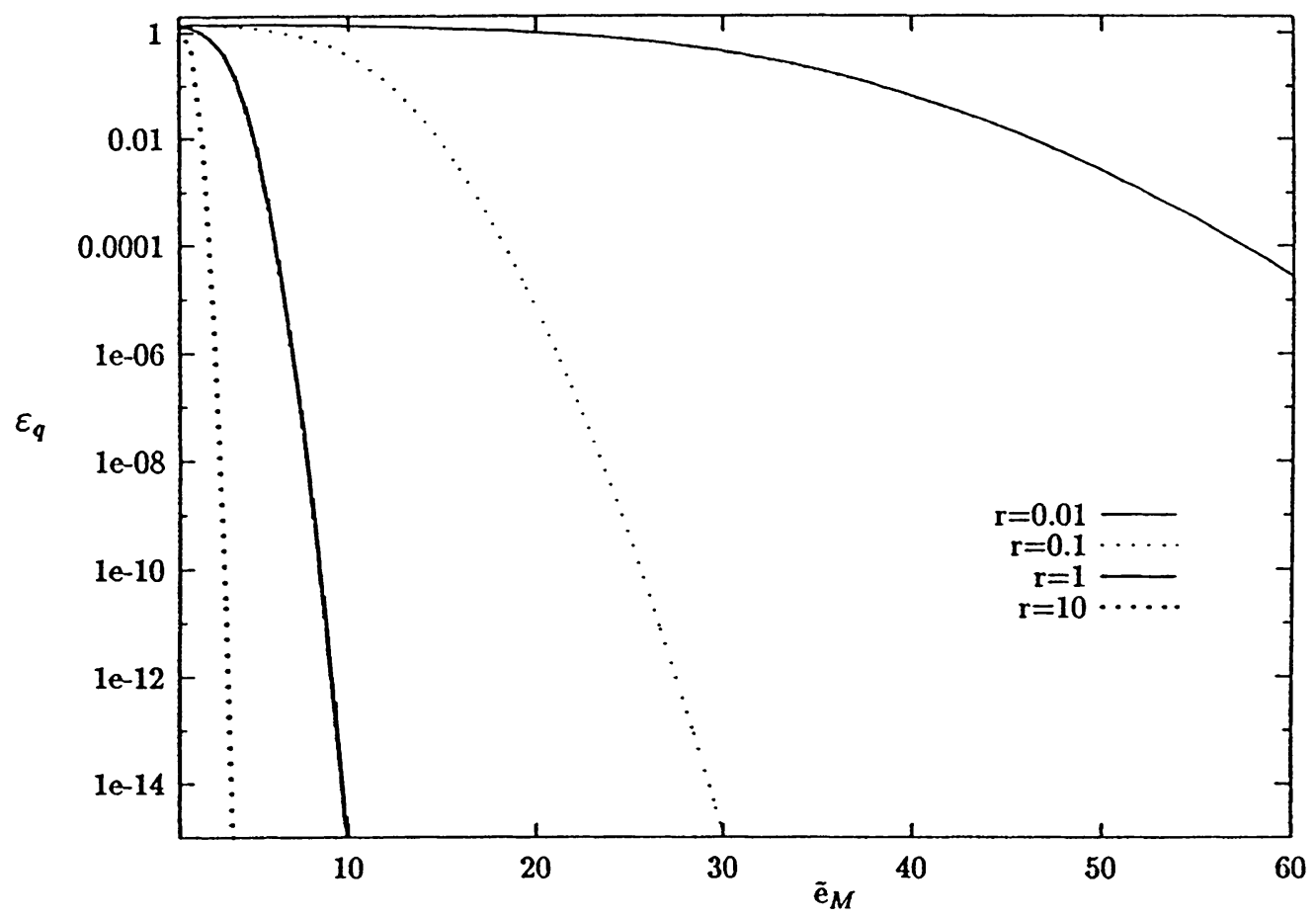

FIG. 2(b). Plot of $\varepsilon_{q}$ as a function of $\tilde{e}_{M}$ at different values of the expected macroscopic parameter $r=3 \rho U^{2} /(2 \varepsilon)$. The plot gives an indication of the value of the parameter $\tilde{e}_{M}$ to be chosen, in the discretization, so that the induced magnitude of the spurious terms is small enough.

\begin{tabular}{|c|c|c|c|c|}
\hline & $\begin{array}{c}\bar{\varepsilon}=10^{-1} \\
\bar{\varepsilon}_{b}=\frac{2}{3} 10^{-1}\end{array}$ & $\begin{array}{c}\bar{\varepsilon}=10^{-2} \\
\bar{\varepsilon}_{b}=\frac{2}{3} 10^{-2}\end{array}$ & $\begin{array}{c}\bar{\varepsilon}=10^{-3} \\
\bar{\varepsilon}_{b}=\frac{2}{3} 10^{-3}\end{array}$ & $\begin{array}{r}\bar{\varepsilon}=10^{-4} \\
\bar{\varepsilon}_{b}=\frac{2}{3} 10^{-4}\end{array}$ \\
\hline$r=10$ & 0.406 & 0.208 & 0.103 & 0.0482 \\
\hline$r=1$ & 1.136 & 0.230 & 0.104 & 0.0466 \\
\hline$r=0.1$ & 2.606 & 0.684 & 0.303 & 0.136 \\
\hline$r=0.01$ & 8.364 & 2.122 & 0.944 & 0.429 \\
\hline
\end{tabular}

TABLE 2. Values of $\bar{e}_{m}\left(r, \bar{\varepsilon}_{m}=\frac{\bar{\varepsilon}}{3}, \bar{\varepsilon}_{M}=\frac{\bar{\varepsilon}}{3}\right)$ defined in (3.19) at different values of the expected macroscopic parameter $r=3 \rho U^{2} /(2 \mathcal{E})$, and of the overall accuracy $\bar{\varepsilon}\left(\bar{\varepsilon}_{b}=\bar{\varepsilon}_{m}+\bar{\varepsilon}_{M}=\frac{2}{3} \bar{\varepsilon}\right)$ and with $\bar{e}_{M}$ given in Table 1. Values above 1 correspond to those cases in which the contribution $\bar{\varepsilon}_{b}$ derived by introducing any nonvanishing physically admissible $\tilde{e}_{m}<1$ is always smaller than required.

Besides, except for very special cases where $\varepsilon_{q}$ is about to change sign (say, magnitude smaller than $\left.10^{-6}\right), \varepsilon_{\pi}$ is always an order of magnitude smaller than $\varepsilon_{q}$. Therefore, $\bar{\varepsilon}_{m}$ and $\bar{\varepsilon}_{M}$ are essentially determined by $\tilde{e}_{m}^{q}$ and $\tilde{e}_{M}^{q}$. As a consequence, if one is not 
concerned with the magnitude of the spurious term in the energy equation of (3.6), but just wants to keep the one in the momentum equation below some magnitude $\alpha$, larger values of $\tilde{e}_{m}$ and smaller values of $\tilde{e}_{M}$ can be used. Roughly, those reported in the columns corresponding to $10 \alpha$ of Tables 1 and 2 . We recall that the dimensional values $e_{m}$ and $e_{M}$ are then given by $e_{m}=U^{2} \tilde{e}_{m}^{2} / 2$ and $e_{M}=U^{2} \tilde{e}_{M}^{2} / 2$.

With this at hand we now describe the discretization procedure.

4. Energy discretization and kinetic model. The criterion discussed above allows us to identify an interval $I_{e}=\left[e_{m}, e_{M}\right)$ such that the probability that a particle has energy outside $I_{e}$ can be considered negligible. This section describes the discretization procedure introduced in $I_{e}$. It consists in dividing $I_{e}$ into $n+1$ subintervals $I_{i}$ of equal width $\Delta$ and in denoting by $e_{i}$ the midpoint of the interval $I_{i}$, that is,

$$
I_{i}=\left[e_{m}+i \Delta, e_{m}+(i+1) \Delta\right), \quad e_{i}=e_{m}+\left(i+\frac{1}{2}\right) \Delta, \quad i=0, \ldots, n,
$$

with

$$
\Delta=\frac{e_{M}-e_{m}}{n+1}
$$

It is trivial to check that

Proposition 4.1. The discretization (4.1), (4.2) has the following properties:

i) $\forall i, j, h$ such that $e_{i}+e_{j}-e_{h} \in I_{e}$, there exists $k=i+j-h, 0 \leq k \leq n$, such that $e_{i}+e_{j}-e_{h}=e_{k}$

ii) if $e_{m}=\ell \Delta, \ell \in \mathbb{N}$, then $\forall i, j$ such that $e_{i}+e_{j} \leq e_{M}$, there exists $K=i+j+\ell$ such that $e_{i}+e_{j}=\sup I_{K}$.

REMARK 4.2. The proposition assures that

i) For any couple of input energies belonging to the discretization, if one of the output energies also belongs to the discretization, then either the other output energy belongs to the discretization, or it is outside $I_{e}$, but, as already stated, the occurrence of this last case has negligible probability.

ii) If $e_{m}=\ell \Delta$, then either $\left[e_{m}, e_{i}+e_{j}\right)$ contains $I_{e}$, or it is exactly equal to the union of $i+j+\ell+1$ subintervals

$$
\left[e_{m}, e_{i}+e_{j}\right)=\bigcup_{h=0}^{i+j+\ell} I_{h} .
$$

This will be important in the next section to properly handle the collision operator. The condition $e_{m}=\ell \Delta$ does not represent a real restriction on the choice of $I_{e}$, since $I_{e}$ can be easily adjusted to satisfy this condition.

The following step consists in considering any function of velocity as a function of energy and velocity direction, and in approximating its energy dependence in $I_{e}$ by a stepwise interpolation defined on the partition $I_{i}$. This means that

$$
F(\mathbf{v})=F(e, \widehat{\mathbf{\Omega}}) \approx \sum_{i=0}^{n} \chi_{i}(e) F_{i}(\widehat{\mathbf{\Omega}})
$$

where $F_{i}(\widehat{\mathbf{\Omega}})=F\left(e_{i}, \widehat{\boldsymbol{\Omega}}\right)$ and $\chi_{i}(e)$ is the characteristic function of the interval $I_{i}$. 
It will be useful to note that since

$$
\int_{I_{i}} \chi_{j}(e) d e=\Delta \delta_{i j}
$$

where $\delta_{i j}$ is the Kronecker delta, one can approximate the integral of a function defined in $I_{e}$ as

$$
\int_{I_{e}} F(e) d e \approx \int_{I_{e}} \sum_{j=0}^{n} \chi_{j}(e) F_{j} d e=\Delta \sum_{j=0}^{n} F_{j}
$$

and

$$
\int_{I_{j}} F(e) d e=\Delta F_{j} .
$$

The quadrature rule (4.6) is nothing else but the midpoint rule which gives an error that goes to zero as $\Delta^{2}$.

As is well known in kinetic theory, the change during the time interval $[t, t+d t]$ of the number of molecules in the element $d \mathbf{x} d \mathbf{v}$ around $(\mathbf{x}, \mathbf{v})$ due to collision is equal to

$J d \mathbf{v} d t d \mathbf{x}=d \mathbf{v} d t d \mathbf{x} \int_{\mathbb{R}^{3} \times S_{2}} \sigma(\gamma, g) g\left[f\left(t, \mathbf{x}, \mathbf{v}^{\prime}\right) f\left(t, \mathbf{x}, \mathbf{v}_{*}^{\prime}\right)-f(t, \mathbf{x}, \mathbf{v}) f\left(t, \mathbf{x}, \mathbf{v}_{*}\right)\right] d \hat{\mathbf{g}}^{\prime} d \mathbf{v}_{*}$,

where $\sigma(\gamma, g)$ is the differential cross section.

Therefore, computing the change during the time interval $[t, t+d t]$ of the number of molecules in the volume element $d \mathbf{x}$ around $\mathbf{x}$, having velocity direction in the element $d \widehat{\boldsymbol{\Omega}}$ around $\widehat{\boldsymbol{\Omega}}$ and energy in $I_{i}$ due to collisions, one has

$$
\begin{array}{r}
\int_{I_{i}} J \sqrt{2 e} d e=\int_{I_{i}} \sqrt{2 e} d e \int_{\mathbb{R}_{+}} \sqrt{2 e_{*}} d e_{*} \int_{S_{2}} d \widehat{\boldsymbol{\Omega}}_{*} \int_{S_{2}} d \hat{\mathbf{g}}^{\prime} \sigma(\gamma, g) g\left[f\left(\mathbf{v}^{\prime}\right) f\left(\mathbf{v}_{*}^{\prime}\right)\right. \\
\left.-f(\sqrt{2 e} \widehat{\boldsymbol{\Omega}}) f\left(\sqrt{2 e_{*}} \widehat{\mathbf{\Omega}}_{*}\right)\right] .
\end{array}
$$

For the purpose of the present paper it is useful to consider the output velocities not as a function of $\hat{\mathbf{g}}^{\prime}$ as in (2.4), but as a function of an output energy $e^{\prime}$ and of the angle $\theta$ between the planes containing the pre- and post-collisional velocities, as reported in the Appendix; see (A.5) and (A.6). This second formulation is preferred because it makes explicit the dependence on the energy $e^{\prime}$ which will then be discretized. This also means that in the scattering cross section, $\gamma$ will be considered as a known function of $e^{\prime}$ and $\theta$ through (A.7).

Referring to Fig. 3 and using Eq. (A.4), one can change the variable in the surface integral over $\hat{\mathbf{g}}^{\prime}$ expressing $d \hat{\mathbf{g}}^{\prime}$ as

$$
d \hat{\mathbf{g}}^{\prime}=\sin \varphi^{\prime} d \varphi^{\prime} d \theta=\frac{4}{g R} d e^{\prime} d \theta
$$

so that (4.9) can be rewritten as

$$
\begin{array}{r}
\int_{I_{i}} J \sqrt{2 e} d e=\int_{I_{i}} d e \int_{\mathbb{R}_{+}} d e_{*} \int_{S_{2}} d \widehat{\boldsymbol{\Omega}}_{*} \int_{\mathcal{D}_{e^{\prime}}} d e^{\prime} \int_{0}^{2 \pi} d \theta \frac{8 \sqrt{e e_{*}}}{R} \sigma(\gamma, g)\left[f\left(\mathbf{v}^{\prime}\right) f\left(\mathbf{v}_{*}^{\prime}\right)\right. \\
\left.-f(\sqrt{2 e} \widehat{\boldsymbol{\Omega}}) f\left(\sqrt{2 e_{*}} \widehat{\boldsymbol{\Omega}}_{*}\right)\right]
\end{array}
$$






FIG. 3. Collision dynamics. Dashed arrows are in the plane of the figure which represents the precollisional plane. Full arrows refer to the post-collisional quantities which are in the plane that intersects the plane of the figure along $\mathbf{R}$ and forms with it an angle $\theta$.

where $\mathcal{D}_{e^{\prime}}$ is given by $(2.13)$ and depends on $e, e_{*}$ and $\widehat{\boldsymbol{\Omega}} \cdot \widehat{\boldsymbol{\Omega}}_{*}$.

In view of the velocity discretization to be done at a later stage, it is convenient to switch the integrations over $\widehat{\boldsymbol{\Omega}}_{*}$ and over $e^{\prime}$. In order to do that, one has to recall what was stated at the end of Sec. 2 , and switch from the classical viewpoint yielding a restriction on the domain of possible post-collisional energies, to that of a restriction on the domain of allowed pre-collisional velocity directions. This allows us to rewrite Eq. (4.11) as

$$
\int_{I_{i}} J \sqrt{2 e} d e=\int_{I_{i}} d e \int_{\mathbb{R}_{+}} d e_{*} \int_{0}^{e+e_{*}} \hat{J}\left(e, e_{*}, e^{\prime}, \widehat{\boldsymbol{\Omega}}\right) d e^{\prime}
$$

where

$$
\begin{aligned}
& \hat{J}\left(e, e_{*}, e^{\prime}, \widehat{\mathbf{\Omega}}\right) \stackrel{\text { def }}{\equiv} \int_{\mathcal{D}_{\boldsymbol{\Omega}_{*}}} d \widehat{\boldsymbol{\Omega}}_{*} \int_{0}^{2 \pi} d \theta \frac{8 \sqrt{e e_{*}}}{R\left(e, e_{*}, \widehat{\boldsymbol{\Omega}} \cdot \widehat{\boldsymbol{\Omega}}_{*}\right)} \sigma\left(e, e_{*}, e^{\prime}, \widehat{\boldsymbol{\Omega}} \cdot \widehat{\boldsymbol{\Omega}}_{*}, \theta\right) \\
& \times\left[f\left(e^{\prime}, \widehat{\boldsymbol{\Omega}}^{\prime}\left(e, e_{*}, e^{\prime}, \widehat{\boldsymbol{\Omega}}, \widehat{\boldsymbol{\Omega}}_{*}, \theta\right)\right) f\left(e_{*}^{\prime}, \widehat{\boldsymbol{\Omega}}_{*}^{\prime}\left(e, e_{*}, e^{\prime}, \widehat{\boldsymbol{\Omega}}, \widehat{\boldsymbol{\Omega}}_{*}, \theta\right)\right)-f(e, \widehat{\boldsymbol{\Omega}}) f\left(e_{*}, \widehat{\boldsymbol{\Omega}}_{*}\right)\right],
\end{aligned}
$$

and $\mathcal{D}_{\hat{\boldsymbol{\Omega}}_{*}}$ is defined by $(2.15)$.

If we approximate the energy dependence of the Boltzmann one-particle distribution function using the stepwise interpolation, and we neglect collisions involving incoming or 
outgoing particles with energies outside $I_{e}$, we get

$$
\begin{aligned}
\int_{I_{i}} J \sqrt{2 e} d e & \approx \sum_{h, j=0}^{n} \int_{I_{i}} \chi_{h}(e) d e \int_{\mathbb{R}_{+}} \chi_{j}\left(e_{*}\right) d e_{*} \int_{0}^{e_{h}+e_{j}} \hat{J}\left(e_{h}, e_{j}, e^{\prime}, \widehat{\boldsymbol{\Omega}}\right) d e^{\prime} \\
& \approx \sum_{h, j=0}^{n} \int_{I_{i}} \chi_{h}(e) d e \int_{\mathbb{R}_{+}} \chi_{j}\left(e_{*}\right) d e_{*} \int_{e_{m}}^{e_{+}^{h}} \hat{J}\left(e_{h}, e_{j}, e^{\prime}, \widehat{\boldsymbol{\Omega}}\right) d e^{\prime} \\
& =\Delta^{2} \sum_{j=0}^{n} \int_{e_{m}}^{e_{+}} \hat{J}\left(e_{i}, e_{j}, e^{\prime}, \widehat{\boldsymbol{\Omega}}\right) d e^{\prime}
\end{aligned}
$$

where $e_{+}^{h}=\min \left\{e_{h}+e_{j}, e_{M}\right\}$ and $e_{+}=\min \left\{e_{i}+e_{j}, e_{M}\right\}$.

Recalling Remark 4.2, and because of Proposition 4.1, the interval of integration over $e^{\prime}$ results either equal to $I_{e}$, or is the union of $i+j+\ell+1$ subintervals of the partition, that is,

$$
e_{+}=\sup I_{h_{+}}, \quad h_{+}=\min \{i+j+\ell, n\} .
$$

Discretizing also $e^{\prime}$ in the now usual fashion gives

$$
\begin{aligned}
\int_{I_{i}} J \sqrt{2 e} d e & \approx \Delta^{2} \sum_{j=0}^{n} \int_{e_{m}}^{e_{+}} \sum_{h=0}^{n} \chi_{h}\left(e^{\prime}\right) d e^{\prime} \hat{J}\left(e_{i}, e_{j}, e_{h}, \widehat{\mathbf{\Omega}}\right) \\
& =\Delta^{3} \sum_{j=0}^{n} \sum_{h=0}^{h_{+}} \hat{J}\left(e_{i}, e_{j}, e_{h}, \widehat{\mathbf{\Omega}}\right) .
\end{aligned}
$$

For consistency, one has also to neglect those collisions having $e_{k}=e_{i}+e_{j}-e_{h}$ outside $I_{e}$. This allows us to write the sum over the index $h$ related to an output particle in a more symmetrical way:

$$
\int_{I_{i}} J \sqrt{2 e} d e \approx \Delta^{3} \sum_{j=0}^{n} \sum_{\substack{h, k=0 \\ h+k=i+j}}^{n} \hat{J}\left(e_{i}, e_{j}, e_{h}, \widehat{\Omega}\right) .
$$

Using the same procedure on the differential operator and equating gives

$$
\frac{\partial f_{i}}{\partial t}=4 \Delta^{2} \sum_{j=0}^{n} \sqrt{2 e_{j}} \sum_{\substack{h, k=0 \\ h+k=i+j}}^{n} \int_{0}^{2 \pi} d \theta \int_{\mathcal{D}_{\widehat{\boldsymbol{\Omega}}_{*}}\left(e_{i}, e_{j}, e_{h}\right)} d \widehat{\boldsymbol{\Omega}}_{*} \frac{\sigma_{i j}^{h k}\left(\widehat{\boldsymbol{\Omega}} \cdot \widehat{\boldsymbol{\Omega}}_{*}, \theta\right)}{R_{i j}\left(\widehat{\boldsymbol{\Omega}} \cdot \widehat{\boldsymbol{\Omega}}_{*}\right)}\left(f_{h}^{\prime} f_{* k}^{\prime}-f_{i} f_{* j}\right)
$$

where

$$
\begin{aligned}
f_{i} & =f_{i}(\widehat{\boldsymbol{\Omega}})=f\left(\sqrt{2 e_{i}} \widehat{\boldsymbol{\Omega}}\right), \\
f_{* j} & =f_{j}\left(\widehat{\boldsymbol{\Omega}}_{*}\right)=f\left(\sqrt{2 e_{j}} \widehat{\boldsymbol{\Omega}}_{*}\right), \\
f_{h}^{\prime} & =f_{h}\left(\widehat{\boldsymbol{\Omega}}_{i j}^{\prime h k}\left(\widehat{\boldsymbol{\Omega}}, \widehat{\boldsymbol{\Omega}}_{*}, \theta\right)\right)=f\left(\sqrt{2 e_{h}} \widehat{\boldsymbol{\Omega}}^{\prime}\left(e_{i}, e_{j}, e_{h}, \widehat{\boldsymbol{\Omega}}, \widehat{\boldsymbol{\Omega}}_{*}, \theta\right)\right), \\
f_{* k}^{\prime} & =f_{k}\left(\widehat{\boldsymbol{\Omega}}_{* i j}^{\prime h k}\left(\widehat{\boldsymbol{\Omega}}, \widehat{\boldsymbol{\Omega}}_{*}, \theta\right)\right)=f\left(\sqrt{2 e_{k}} \widehat{\boldsymbol{\Omega}}_{*}^{\prime}\left(e_{i}, e_{j}, e_{h}, \widehat{\boldsymbol{\Omega}}, \widehat{\boldsymbol{\Omega}}_{*}, \theta\right)\right),
\end{aligned}
$$


and where $\sigma_{i j}^{h k}, R_{i j}, \widehat{\boldsymbol{\Omega}}_{i j}^{\prime h k}$, and $\widehat{\boldsymbol{\Omega}}_{* i j}^{\prime h k}$ are respectively the differential cross section (see also (A.7)), the collision momentum, and the post-collisional directions given in (A.6) computed for $e=e_{i}, e_{*}=e_{j}, e^{\prime}=e_{h}$, and $e_{*}^{\prime}=e_{k}$.

REMARK 4.3. The integrand in (4.18) presents a denominator that vanishes in the case of head-on collisions $e_{i}=e_{j}, \widehat{\boldsymbol{\Omega}}=-\widehat{\boldsymbol{\Omega}}_{*}$, i.e., for $R=0$. Thus, we have to check whether the integrand has a singularity in that circumstance. Referring to Fig. 1 and choosing $\widehat{\boldsymbol{\Omega}}$ as polar axis in the integration over $\mathcal{D}_{\widehat{\boldsymbol{\Omega}}_{*}}$, one has that

$$
\frac{\sqrt{2 e_{i}} d \widehat{\boldsymbol{\Omega}}_{*}}{R_{i i}\left(\widehat{\boldsymbol{\Omega}} \cdot \widehat{\boldsymbol{\Omega}}_{*}\right)}=\frac{\sin \beta d \beta d \Gamma}{\sqrt{2\left(1+\widehat{\boldsymbol{\Omega}} \cdot \widehat{\boldsymbol{\Omega}}_{*}\right)}}=\frac{\sin \frac{\beta}{2} \cos \frac{\beta}{2}}{\sqrt{\frac{1+\cos \beta}{2}}} d \beta d \Gamma=\sin \frac{\beta}{2} d \beta d \Gamma,
$$

where $\Gamma$ is the longitude and $\beta$ the colatitude $\left(\cos \beta=\widehat{\Omega} \cdot \widehat{\boldsymbol{\Omega}}_{*}\right)$, because $e_{i}=e_{j}$. Therefore, there is no singularity as $\beta$ goes to $\pi$.

If one rewrites the kinetic model (4.18) in terms of velocity moduli instead of energies of the particles, then it is possible to realize that it falls into the class of models studied in [8]. Hence, the following theorem holds.

Theorem 4.4. The kinetic model (4.18) has the following properties.

i) It preserves mass, momentum, and energy, defined by

$$
\begin{aligned}
\rho_{d} & =m \Delta \sum_{j=0}^{n} \sqrt{2 e_{j}} \int_{\mathbf{S}_{2}} f_{j}(\widehat{\boldsymbol{\Omega}}) d \widehat{\boldsymbol{\Omega}}, \\
\mathbf{U}_{d} & =\frac{2 m \Delta}{\rho_{d}} \sum_{j=0}^{n} e_{j} \int_{\mathbf{S}_{2}} \widehat{\boldsymbol{\Omega}} f_{j}(\widehat{\boldsymbol{\Omega}}) d \widehat{\boldsymbol{\Omega}}, \\
\mathcal{E}_{d} & =\frac{m \Delta}{2} \sum_{j=0}^{n} \sqrt{2 e_{j}} \int_{\mathbf{S}_{2}}\left|\sqrt{2 e_{j}} \widehat{\boldsymbol{\Omega}}-\mathbf{U}\right|^{2} f_{j}(\widehat{\boldsymbol{\Omega}}) d \widehat{\boldsymbol{\Omega}} .
\end{aligned}
$$

ii) In the nonhomogeneous case, it yields the usual conservation equations:

$$
\begin{aligned}
& \frac{\partial \rho_{d}}{\partial t}+\nabla \cdot\left(\rho_{d} \mathbf{U}_{d}\right)=0, \\
& \frac{\partial}{\partial t}\left(\rho_{d} \mathbf{U}_{d}\right)+\nabla \cdot\left(\mathbf{\Pi}_{d}+\rho_{d} \mathbf{U}_{d} \otimes \mathbf{U}_{d}\right)=\mathbf{0} \\
& \frac{\partial}{\partial t}\left(\mathcal{E}_{d}+\frac{1}{2} \rho_{d} U_{d}^{2}\right)+\nabla \cdot\left[\mathbf{q}_{d}+\boldsymbol{\Pi}_{d} \mathbf{U}_{d}+\left(\mathcal{E}_{d}+\frac{1}{2} \rho_{d} U_{d}^{2}\right) \mathbf{U}_{d}\right]=0,
\end{aligned}
$$

where

$$
\begin{gathered}
\boldsymbol{\Pi}_{d}=m \Delta \sum_{j=0}^{n} \sqrt{2 e_{j}} \int_{\mathbf{S}_{2}}\left(\sqrt{2 e_{j}} \widehat{\mathbf{\Omega}}-\mathbf{U}\right) \otimes\left(\sqrt{2 e_{j}} \widehat{\boldsymbol{\Omega}}-\mathbf{U}\right) f_{j}(\widehat{\boldsymbol{\Omega}}) d \widehat{\boldsymbol{\Omega}} \\
\mathbf{q}_{d}=\frac{m \Delta}{2} \sum_{j=0}^{n} \sqrt{2 e_{j}} \int_{\mathbf{S}_{2}}\left|\sqrt{2 e_{j}} \widehat{\mathbf{\Omega}}-\mathbf{U}\right|^{2}\left(\sqrt{2 e_{j}} \widehat{\boldsymbol{\Omega}}-\mathbf{U}\right) f_{j}(\widehat{\boldsymbol{\Omega}}) d \widehat{\boldsymbol{\Omega}}
\end{gathered}
$$

iii) The Maxwellian equilibrium state is defined by the following equivalent conditions:

(a) $\left(\log f_{0}, \ldots, \log f_{n}\right)$ belongs to the space of collisional invariant;

(b) $f_{i}=A \exp \left[\sqrt{b e_{i}} \hat{\mathbf{b}} \cdot \widehat{\mathbf{\Omega}}-c e_{i}\right], \quad A>0$; 
(c) $\mathbf{J}[\mathbf{f}, \mathbf{f}]=\mathbf{0}$ where $\mathbf{f}=\left(f_{0}, \ldots, f_{n}\right)$ and $\mathbf{J}=\left(J_{0}, \ldots, J_{n}\right)$ is the right-hand side of (4.18).

iv) In the spatially homogeneous case, the Boltzmann $H$-functional

$$
H=\sum_{i=0}^{n} \sqrt{2 e_{i}} \int_{\mathbf{S}_{2}} f_{i} \log f_{i} d \widehat{\mathbf{\Omega}},
$$

is such that $d H / d t \leq 0$ where the equality sign holds if and only if the system is in the Maxwellian equilibrium state.

It has to be observed that the macroscopic quantities defined in (4.21) and (4.23) related to the discretization are the consistent approximation of the corresponding quantities related to the continuous Boltzmann equation through the now usual approximation procedure.

The relation between the Maxwellian parameters and the definition of the macroscopic observables $\rho_{d}, \mathbf{U}_{d}$, and $e_{d}$ can be obtained by substituting in (4.21) the Maxwellian distribution densities. After some computation, one obtains

$$
\begin{aligned}
\rho_{d} & =4 \pi m A \Delta \sqrt{\frac{2}{b}} \sum_{i=0}^{n} e^{-c e_{i}} \sinh \sqrt{b e_{i}} \\
\rho_{d} U_{d} & =\frac{8 \pi m A \Delta}{b} \sum_{i=0}^{n} e^{-c e_{i}}\left(\sqrt{b e_{i}} \cosh \sqrt{b e_{i}}-\sinh \sqrt{b e_{i}}\right) \\
\widehat{\mathbf{U}}_{d} & =\hat{\mathbf{b}} \\
\mathcal{E}_{d}+\frac{1}{2} \rho_{d} U_{d}^{2} & =4 \pi m A \Delta \sqrt{\frac{2}{b}} \sum_{i=0}^{n} e_{i} e^{-c e_{i}} \sinh \sqrt{b e_{i}}
\end{aligned}
$$

where the subscript $d$ stresses the fact that these macroscopic quantities are computed from the discretized model and the hats denote unit vectors.

As for discrete models, the map (4.25) is one-to-one, even though the inversion cannot in general be performed in terms of elementary functions.

If one writes the Euler equations related to the kinetic model (4.18), then again equations similar to (3.6) are found, where the macroscopic observables are replaced by their discretized version, and where $\varepsilon_{\pi}$ and $\varepsilon_{q}$ now become

$$
\begin{gathered}
\varepsilon_{\pi}^{d}=\frac{\sum_{i, j=0}^{n} e^{-c\left(e_{i}+e_{j}\right)}\left[\left(b e_{i}+2\right) \sinh \sqrt{b e_{i}} \sinh \sqrt{b e_{j}}-b \sqrt{e_{i} e_{j}} \cosh \sqrt{b e_{i}} \cosh \sqrt{b e_{j}}-\sqrt{b e_{i}} \cosh \sqrt{b e_{i}} \sinh \sqrt{b e_{j}}\right]}{\left[\sum_{i=0}^{n} e^{-c e_{i}}\left(\sqrt{b e_{i}} \cosh \sqrt{b e_{i}}-\sinh \sqrt{b e_{i}}\right)\right]^{2}}, \\
\left.\varepsilon_{q}^{d}=\frac{\sum_{i, j=0}^{n} e^{-c\left(e_{i}+e_{j}\right)}\left(e_{i}-e_{j}\right) \sqrt{e_{i}} \cosh \sqrt{b e_{i}} \sinh \sqrt{b e_{j}}}{2\left[\sum_{i=0}^{n} e^{-c e_{i}}\left(\sqrt{b e_{i}} \cosh \sqrt{b e_{i}}-\sinh \sqrt{b e_{i}}\right)\right]^{2}} b \sqrt{b}-1.26\right)
\end{gathered}
$$

With respect to the spurious terms $\varepsilon_{\pi}$ and $\varepsilon_{q}$ encountered in Sec. 3, when limiting the range of allowed energies, $\varepsilon_{\pi}^{d}$ and $\varepsilon_{q}^{d}$ present a new contribution related to the discretization of the interval $\left[e_{m}, e_{M}\right]$ into $n+1$ subintervals and to the introduction of the midpoint rule, which brings an error of order $\Delta^{2}$. Actually, the constant of proportionality could be explicitly written down, but it is useless, since what is important is the 
overall magnitude of the spurious terms, which is expressed by (4.26) and (4.27), rather than partial information on the last discretization step.

It should be noticed that $\varepsilon_{\pi}^{d}$ and $\varepsilon_{q}^{d}$ depend on the Maxwellian parameters $b$ and $c$, which are related to the macroscopic observables through (4.27), and not through (3.2). In more detail, their dimensionless values $\tilde{b}=\sqrt{\frac{b}{2}} U$ and $\tilde{c}=c U^{2}$ are determined by

$$
\left\{\begin{array}{l}
\sum_{i=0}^{n} e^{-\tilde{c} \tilde{e}_{i}^{2} / 2}\left[\tilde{b} \tilde{e}_{i} \cosh \tilde{b} \tilde{e}_{i}-(\tilde{b}+1) \sinh \tilde{b} \tilde{e}_{i}\right]=0 \\
\sum_{i=0}^{n} e^{-\tilde{c} \tilde{e}_{i}^{2} / 2}\left(\tilde{e}_{i}-1-\frac{3}{r}\right) \sinh \tilde{b} \tilde{e}_{i}=0
\end{array}\right.
$$

However, for not too small values of $n$, we have $\tilde{b} \approx \tilde{c} \approx r$. Moreover, using the values obtained by solving (4.28) or $\tilde{b}=\tilde{c}=r$ gives no or very little difference in the identification of the discretization parameter $n$ through the procedure described below.

Having chosen the discretization interval as explained in Sec. 3, i.e., $\bar{\varepsilon}_{M I}$ and $\bar{\varepsilon}_{m}$ defined by (3.18) and (3.19), respectively, through $\bar{\varepsilon}_{M}$ and $\bar{\varepsilon}_{m}$, and the desired accuracy $\bar{\varepsilon}_{n}$ for this discretization, one can set $n$ (which will be of order $1 / \sqrt{\bar{\varepsilon}_{n}}$ ) such that the overall discretization terms defined in Eqs. (4.26) and (4.27) are both smaller than $\bar{\varepsilon}=\bar{\varepsilon}_{M}+$ $\bar{\varepsilon}_{m}+\bar{\varepsilon}_{n}$, that is,

$$
\varepsilon^{d}\left(\tilde{e}_{m}, \tilde{e}_{M}, n\right) \stackrel{\text { def }}{\equiv} \max \left\{\left|\varepsilon_{\pi}^{d}\right|,\left|\varepsilon_{q}^{d}\right|\right\}<\bar{\varepsilon} .
$$

The results of such a procedure are reported in Table 3 where the value of $n$ determined for different values of $r$ and accuracy $\bar{\varepsilon}$ with $\bar{\varepsilon}_{M}=\bar{\varepsilon}_{m}=\bar{\varepsilon}_{n}=\frac{\bar{\varepsilon}}{3}$ is reported.

$$
\begin{aligned}
& r=10 \\
& r=1 \\
& r=1 \\
& r=0.1 \\
& r=0.01
\end{aligned} \quad\left(\begin{array}{cccc}
\bar{\varepsilon}=10^{-1} & \bar{\varepsilon}=10^{-2} & \bar{\varepsilon}=10^{-3} & \bar{\varepsilon}=10^{-4} \\
2 & 14 & 52 & 238 \\
7 & 10 & 250 & 1451 \\
5 & 9 & 263 & 1531 \\
5 & 9 & 350 & 1524
\end{array}\right)
$$

TABLE 3. Values of $n$ at different values of $r$, such that the spurious terms defined in (4.26) and (4.27) are definitely smaller than the given accuracy $\bar{\varepsilon}$. The values of $\bar{e}_{M}$ and $\bar{e}_{m}$ are those obtained in Tables 1 and 2 , corresponding to $\bar{\varepsilon}_{M}=\bar{\varepsilon}_{m}=\bar{\varepsilon}_{n}=\frac{\bar{\varepsilon}}{3}$. In those cases in which any value of $\tilde{e}_{m} \leq 0.5$ gave a contribution smaller than $\bar{\varepsilon} / 3$ the value $\tilde{e}_{m}=0.5$ has been fixed.

The number of discrete energies needed to keep the spurious terms below a moderate magnitude is very small, but rapidly grows if greater accuracy is required. In Table 4 the same thing is repeated with the values of $\tilde{e}_{M}$ given in Table 1 and with $\tilde{e}_{m}=0$. In this way the effects of the introduction of a nonvanishing $\tilde{e}_{m}$ are evidenced. In our opinion, to take $\tilde{e}_{m} \neq 0$ is worthwhile only for very small $\bar{\varepsilon}$, while due to the faster decay of the contribution related to the choice of $\tilde{e}_{M}$, the possibility of setting $\bar{\varepsilon}_{n}>\bar{\varepsilon}_{M}$ might be of interest. 


$$
\begin{aligned}
& r=10 \\
& r=1 \\
& r=1 \\
& r=0.1 \\
& r=0.01
\end{aligned} \quad\left(\begin{array}{cccc}
\bar{\varepsilon}=10^{-1} & \bar{\varepsilon}=10^{-2} & \bar{\varepsilon}=10^{-3} & \bar{\varepsilon}=10^{-4} \\
5 & 17 & 70 & 344 \\
5 & 9 & 391 & 2208 \\
5 & 9 & 411 & 2330 \\
5 & 9 & 413 & 2299
\end{array}\right)
$$

TABLE 4. Values of $n$ at different values of $r$, such that the spurious terms defined in (4.26) and (4.27) are definitely smaller than the given accuracy $\bar{\varepsilon}$. The values of $\bar{e}_{M}$ are those obtained in Table 1, while $\tilde{e}_{m}$ is always set to zero.

The values reported in Tables 3 and 4 are computed starting from large values of $n$, which, of course, yield an overall magnitude of the spurious terms $\varepsilon^{d} \approx \bar{\varepsilon}_{m}+\bar{\varepsilon}_{M}$, and reporting the value for which the value $\bar{\varepsilon}$ is achieved. However, it should be noticed that the terms due to the three steps of the discretization often do not have the same sign. Therefore, for given $\tilde{e}_{m}$ and $\tilde{e}_{M}$, it is possible to find much smaller values of $n$ for which the overall magnitude $\varepsilon^{d}$ almost vanishes. This behavior is evidentiated in Fig. 4 where the cusps in the log scales locate the change of sign. Typically, $\varepsilon^{d}$ is negative for very large values of $n$, then becomes positive, and then again negative at very small values of $n$. In this last case, because of the poor resolution, the change of sign may generate in the log-scale an abrupt change of slope, rather than a cusp. An example of smaller values of $n$ yielding a given overall magnitude of $\varepsilon$ is reported in Table 5 .

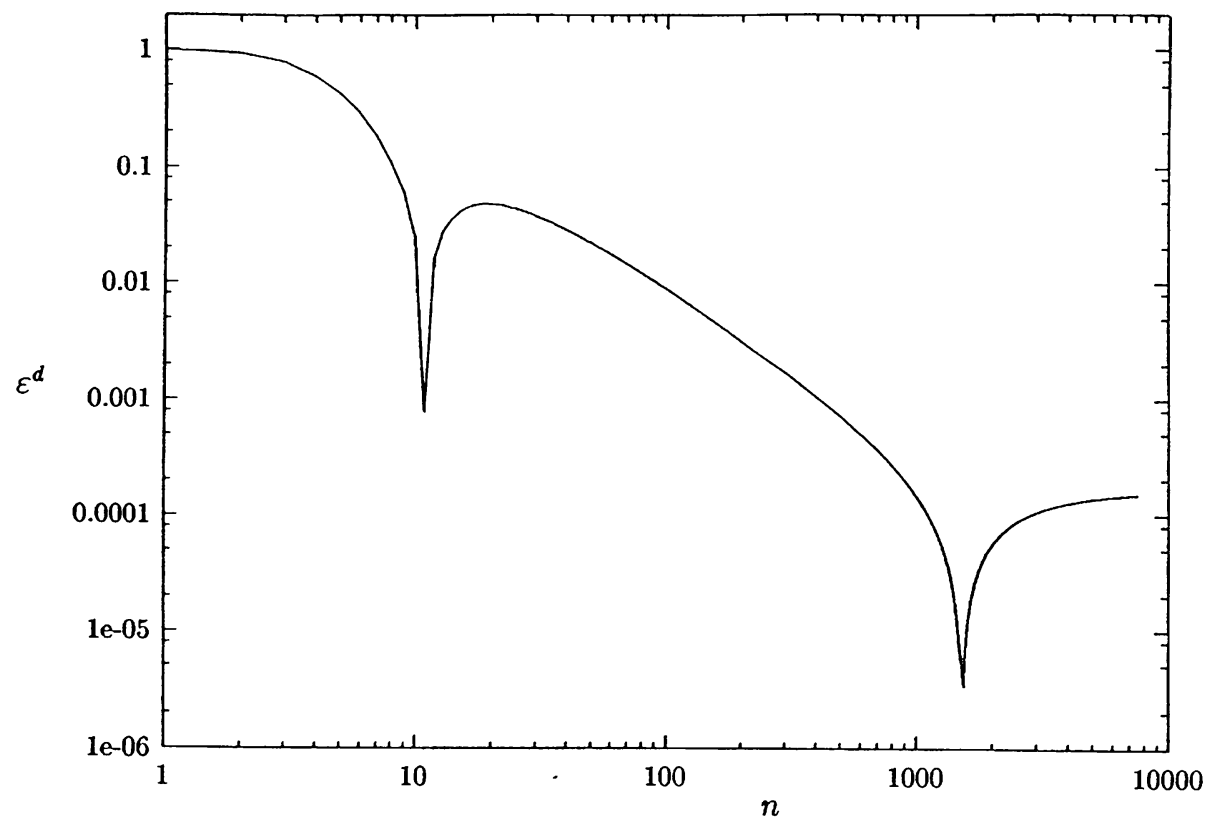

Fig. 4. Plot of $\varepsilon^{d}$ as a function of $n$ for $\tilde{e}_{m}=0, \tilde{e}_{M}=17.49$, and $r=0.1$. The non-regular minima correspond to change of sign in $\varepsilon^{d}$. Notice the asymptotic behaviour towards the value $\bar{\varepsilon}_{b}=\bar{\varepsilon}_{M}=$ $\frac{1}{3} 10^{-3}$ determined when $\mathbb{R}_{+}$is substituted with $[0,17.49]$. 
The application of the kinetic model and of the identification method presented here to some relaxation tests is currently under study.

$$
\begin{aligned}
& \bar{\varepsilon}=10^{-1} \quad \bar{\varepsilon}=10^{-2} \quad \bar{\varepsilon}=10^{-3} \quad \bar{\varepsilon}=10^{-4} \\
& \begin{array}{l}
r=10 \\
r=1 \\
r=0.1
\end{array} \quad\left(\begin{array}{cccc}
(0,2.1,5) & (0.39,2.3,7) & (0.3,2.46,15) & (0.21,2.63,33) \\
(0,4.7,5) & (0.1,5.4,9) & (0.35,5.92,22) & (0.27,6.45,72) \\
(0,14,5) & (0,16,9) & (0.71,17.5,13) & (0.5,19.1,208)
\end{array}\right)
\end{aligned}
$$

TABLE 5. Examples of values of $\left(\tilde{e}_{m}, \tilde{e}_{M}, n\right)$ with $n$ smaller than the value reported in Table 4 and which yield the desired overall magnitude of the spurious terms.

5. Conclusions. We have deduced a kinetic model based on a discretization procedure of the Boltzmann equation written in the energy formulation, in which particles can take a discrete number of equidistributed energies, and which has the property to preserve automatically mass, momentum, and energy.

One of the advantages of this formulation is that for given pre-collisional energies, conservation of energy is satisfied naturally by properly choosing the post-collisional energy (i.e., the velocities on suitable spheres). Then conservation of momentum for fixed pre-collisional velocities and post-collisional energies restricts the post-collisional velocities to be on given (symmetric) parallels of the sphere with the polar axis along the collision momentum $\mathbf{R}$, with center in $\mathbf{R} / 2$, and with diameter equal to the modulus of the relative velocity.

In place of the five-fold collision integral over an infinite domain, the discretized model is characterized by a collision term which consists of a sum of integrals over finite domains, namely the cartesian product of a unit circle and the portion of the unit sphere between two parallels symmetric with respect to the equatorial plane perpendicular to the velocity of the field particle, which can be performed with good precision and small computational effort.

Since the model has been obtained via a controllable approximation procedure, it is possible to obtain estimates on the "distance" between the discretized collision operator and the continuous Boltzmann equation, i.e., consistency of the quadrature rule. Furthermore, there exists an $H$-functional describing trend towards equilibrium described by a Gaussian distribution. Finally, the fluid-dynamic limit related to the discretized Boltzmann equation tends rapidly towards the usual Euler equations with an isotropic pressure tensor and vanishing heat flux, when the number of energies tends to infinity and the discretization interval tends to $\mathbb{R}_{+}$.

Actually, the evaluation of the magnitude of the spurious terms gives a procedure to set the discretization parameters $e_{m}, e_{M}$, and $n$, which then determine the values of discretized energies $e_{i}=e_{m}+\left(e_{M}-e_{m}\right)\left(i+\frac{1}{2}\right) /(n+1)$ for $i=0, \ldots, n$.

Particular attention is given in determining these parameters as a function of the macroscopic characteristics of the fluid-dynamic problem to be studied. The application of the kinetic model and of the identification procedure to problems of fluid-dynamic interest is currently under study. 
Acknowledgments. Partially supported by the Ministry for Scientific Research and by the National Council for Research, C.N.R.-G.N.F.M.

Appendix: Energy formulation of the collision dynamics. Considering a particle as characterized by its energy per unit mass, $e=v^{2} / 2$, and by its velocity direction, one has that the pre- and post-collisional energies, denoted respectively by $\left(e, e_{*}\right)$ and $\left(e^{\prime}, e_{*}^{\prime}\right)$, are related through energy conservation

$$
E \stackrel{\text { def }}{\equiv} e+e_{*}=e^{\prime}+e_{*}^{\prime}
$$

while the pre- and post-collisional directions, denoted respectively by $\left(\widehat{\boldsymbol{\Omega}}, \widehat{\boldsymbol{\Omega}}_{*}\right)$ and $\left(\widehat{\boldsymbol{\Omega}}^{\prime}, \widehat{\boldsymbol{\Omega}}_{*}^{\prime}\right)$, are related through the conservation of momentum, and are given, up to a scattering angle, by

$$
\begin{aligned}
& \widehat{\boldsymbol{\Omega}}^{\prime}=\frac{1}{2 \sqrt{e^{\prime}}}\left(\sqrt{e} \widehat{\boldsymbol{\Omega}}+\sqrt{e_{*}} \widehat{\boldsymbol{\Omega}}_{*}+\sqrt{E-S} \hat{\mathbf{g}}^{\prime}\right) \\
& \widehat{\boldsymbol{\Omega}}_{*}^{\prime}=\frac{1}{2 \sqrt{e_{*}^{\prime}}}\left(\sqrt{e} \widehat{\boldsymbol{\Omega}}+\sqrt{e_{*}} \widehat{\boldsymbol{\Omega}}_{*}-\sqrt{E-S} \hat{\mathbf{g}}^{\prime}\right),
\end{aligned}
$$

where $\hat{\mathbf{g}}^{\prime} \in \mathbf{S}_{2}$, and $S$ is defined in (2.7).

To clarify the relation between the incoming and the outgoing velocity direction, it is useful to refer to Fig. 3 and to decompose the direction of the pre- and post-collisional relative velocities $\hat{\mathbf{g}}$ and $\hat{\mathbf{g}}^{\prime}$ in a component along $\mathbf{R}$, and in another component orthogonal to it:

$$
\begin{gathered}
\hat{\mathbf{g}}=\cos \varphi \widehat{\mathbf{R}}+\sin \varphi \hat{\mathbf{e}}_{R}, \\
\hat{\mathbf{g}}^{\prime}=\cos \varphi^{\prime} \widehat{\mathbf{R}}+\sin \varphi^{\prime} \hat{\mathbf{e}}_{R}^{\prime},
\end{gathered}
$$

where the unit vectors $\hat{\mathbf{e}}_{R}$ and $\hat{\mathbf{e}}_{R}^{\prime}$ are orthogonal to $\widehat{\mathbf{R}}=\mathbf{R} /|\mathbf{R}|$ and belong, respectively, to the plane containing the pre- and post-collisional directions.

The expression of the angles $\varphi \in[0, \pi]$ and $\varphi^{\prime} \in[0, \pi]$ in (A.3) formed by $\hat{\mathbf{g}}$ and $\widehat{\mathbf{R}}$ and by $\hat{\mathbf{g}}^{\prime}$ and $\widehat{\mathbf{R}}$ respectively, is given by

$$
\begin{aligned}
\cos \varphi & =\hat{\mathbf{g}} \cdot \widehat{\mathbf{R}}=\frac{2\left(e_{*}-e\right)}{g R}=\frac{E-2 e}{\sqrt{E^{2}-S^{2}}} \Rightarrow \sin \varphi=\sqrt{\frac{4 e e_{*}-S^{2}}{E^{2}-S^{2}}} \\
\cos \varphi^{\prime} & =\hat{\mathbf{g}}^{\prime} \cdot \widehat{\mathbf{R}}=\frac{2\left(e^{\prime}-e_{*}^{\prime}\right)}{g R}=\frac{2 e^{\prime}-E}{\sqrt{E^{2}-S^{2}}} \Rightarrow \sin \varphi^{\prime}=\sqrt{\frac{4 e^{\prime} e_{*}^{\prime}-S^{2}}{E^{2}-S^{2}}}
\end{aligned}
$$

where $R=|\mathbf{R}|$, while the unit vector $\hat{\mathbf{e}}_{R}^{\prime}$ can, for instance, be defined by

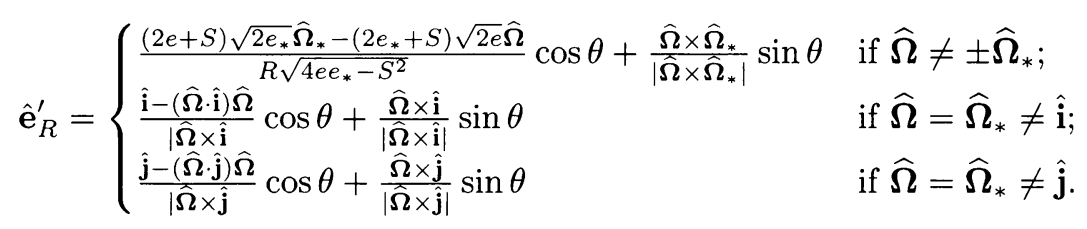

The angle $\theta \in[0,2 \pi)$ appearing in (A.5) is the angle formed by the pre- and postcollisional plane and is not determined by the conservation laws. 
In conclusion, the outgoing velocity directions can be written as

$$
\begin{aligned}
& \widehat{\boldsymbol{\Omega}}^{\prime}=\frac{\sqrt{4 e^{\prime} e_{*}^{\prime}-S^{2}}}{\sqrt{2 e^{\prime}} R} \hat{\mathbf{e}}_{R}^{\prime}+\frac{2 e^{\prime}+S}{\sqrt{e^{\prime}} R^{2}}\left(\sqrt{e} \widehat{\boldsymbol{\Omega}}+\sqrt{e_{*}} \widehat{\boldsymbol{\Omega}}_{*}\right), \\
& \widehat{\boldsymbol{\Omega}}_{*}^{\prime}=-\frac{\sqrt{4 e^{\prime} e_{*}^{\prime}-S^{2}}}{\sqrt{2 e_{*}^{\prime}} R} \hat{\mathbf{e}}_{R}^{\prime}+\frac{2 e_{*}^{\prime}+S}{\sqrt{e_{*}^{\prime}} R^{2}}\left(\sqrt{e} \widehat{\boldsymbol{\Omega}}+\sqrt{e_{*}} \widehat{\boldsymbol{\Omega}}_{*}\right) .
\end{aligned}
$$

From Eq. (2.4) and Fig. 3 it is evident that, as $\hat{\mathbf{g}}^{\prime}$ runs over $\mathbf{S}_{2}$, the post-collisional velocities $\mathbf{v}^{\prime}$ and $\mathbf{v}_{*}^{\prime}$ span the surface of the sphere centered in $\mathbf{R} / 2$ with diameter equal to the modulus of the relative velocity $g$. Furthermore, for any $\hat{\mathbf{g}}^{\prime}$ the post-collisional velocities point at two antipodal points. In more detail, if the polar axis of the unit sphere is set along $\mathbf{R}$, as $\theta$ varies, one of the post-collisional velocities runs over a given northern parallel and the other over the relative southern parallel (at the antipodes). The other angle defining $\hat{\mathbf{g}}^{\prime}$, the colatitude $\varphi^{\prime}$, determines through (A.4) the energy of the outgoing particle.

As is well known, the scattering cross section is a function of the modulus of the relative velocity and of the deflection angle $\alpha \in[0, \pi]$ formed by the pre- and postcollisional relative velocities, which can be written as

$$
\begin{aligned}
\gamma \stackrel{\text { def }}{=} \cos \alpha & =\hat{\mathbf{g}} \cdot \hat{\mathbf{g}}^{\prime}=\cos \varphi \cos \varphi^{\prime}+\sin \varphi \sin \varphi^{\prime} \cos \theta \\
& =\frac{1}{E^{2}-S^{2}}\left\{\left(e_{*}-e\right)\left(e^{\prime}-e_{*}^{\prime}\right)+4 e e_{*} \sqrt{\left[1-\left(\widehat{\boldsymbol{\Omega}} \cdot \widehat{\boldsymbol{\Omega}}_{*}\right)^{2}\right]\left[\lambda-\left(\widehat{\boldsymbol{\Omega}} \cdot \widehat{\boldsymbol{\Omega}}_{*}\right)^{2}\right]} \cos \theta\right\},
\end{aligned}
$$

where $\lambda$ is defined in (2.12).

Note that $\gamma$ is an even function of $\widehat{\boldsymbol{\Omega}} \cdot \widehat{\boldsymbol{\Omega}}_{*}$ and of $\theta$, and observe that

$$
\begin{aligned}
\gamma\left(e_{*}, e, e_{*}^{\prime}, \widehat{\boldsymbol{\Omega}}_{*} \cdot \widehat{\boldsymbol{\Omega}}, \theta\right) & =\gamma\left(e, e_{*}, e^{\prime}, \widehat{\boldsymbol{\Omega}} \cdot \widehat{\boldsymbol{\Omega}}_{*}, \theta\right), \\
\gamma\left(e^{\prime}, e_{*}^{\prime}, e, \widehat{\boldsymbol{\Omega}}^{\prime} \cdot \widehat{\boldsymbol{\Omega}}_{*}^{\prime},-\theta\right) & =\gamma\left(e, e_{*}, e^{\prime}, \widehat{\boldsymbol{\Omega}} \cdot \widehat{\boldsymbol{\Omega}}_{*}, \theta\right),
\end{aligned}
$$

which means that the deflection angle does not change, neither if we exchange the role of the field and test particles, nor in the inverse transformation. In the paper the scattering cross section is then considered as a function of $e, e_{*}, e^{\prime}, \widehat{\boldsymbol{\Omega}} \cdot \widehat{\boldsymbol{\Omega}}_{*}$, and $\theta$.

\section{REFERENCES}

[1] H. Neunzert and J. Struckmeier, Particle methods for the Boltzmann equation, Acta Numerica, 1995, Cambridge University Press, Cambridge, 1995, pp. 417-457

[2] V. V. Aristov and F. G. Tcheremissine, The conservative splitting method for solving Boltzmann's equation, USSR Comp. Math. Phys. 21, 208-225 (1980)

[3] C. Buet, $A$ discrete-velocity scheme for the Boltzmann operator of rarefied gas-dynamics, in Proceedings of 19th Rarefied Gas Dynamics Symposium, J. Harvey and G. Lord, eds., Oxford University Press, 1994, pp. 878-884

[4] C. Buet, A discrete-velocity scheme for the Boltzmann operator of rarefied gas dynamics, Transport Theory Statist. Phys. 25, 33-60 (1996)

[5] A. V. Bobylev, A. Palczewski, and J. Schneider, A consistency result for a discrete-velocity model of the Boltzmann equation, SIAM J. Numer. Anal. 34, 1865-1883 (1997)

[6] F. Rogier and J. Schneider, A direct method for solving the Boltzmann equation, Transport Theory Statist. Phys. 23, 313-338 (1994)

[7] F. G. Tcheremissine, Conservative discrete ordinates method for solving Boltzmann kinetic equation, Comm. Appl. Math. (1997). In press. 
[8] L. Preziosi and E. Longo, On a conservative polar discretization of the Boltzmann equation, Japan J. Indust. Appl. Math. 14, 399-435 (1997)

[9] R. Temam, Sur la stabilité et la convergence de la method des pas fractionaires, Ann. Math. Pura Appl. 79, 191-380 (1968)

[10] L. Desvillettes and S. Mischler, About the splitting algorithm for Boltzmann and B.G.K. equations, Math. Models Methods Appl. Sci. 6, 1079-1101 (1996)

[11] R. J. Di Perna and P.-L. Lions, On the Cauchy problem for Boltzmann equations: Global existence and weak stability, Ann. Math. 130, 321-366 (1989) 